\title{
Compressive Classification for TEM-EELS
}

\author{
Weituo Hao ${ }^{1}$, Andrew Stevens ${ }^{1,2}$, Hao Yang ${ }^{3}$, Michael Gehm², Nigel D. Browning ${ }^{1,4}$ \\ 1. Pacific Northwest National Laboratory, Richland, WA USA. \\ 2. Duke University, Electrical and Computer Engineering, Durham, NC USA. \\ 3. Lawrence Berkeley National Laboratory, Berkeley, CA USA. \\ 4. University of Washington, Materials Science and Engineering, Seattle, WA USA
}

Electron energy loss spectroscopy (EELS) is typically conducted in STEM mode with a spectrometer, or in TEM mode with energy selection. These methods produce a 3D data set ( $x, y$, energy). Some compressive sensing [1,2] and inpainting [3,4,5] approaches have been proposed for recovering a full set of spectra from compressed measurements. In many cases the final form of the spectral data is an elemental map (an image with channels corresponding to elements). This means that most of the collected data is unused or summarized. We propose a method to directly recover the elemental map with reduced dose and acquisition time.

We have designed a new computational TEM sensor for compressive classification [6,7] of energy loss spectra called TEM-EELS. A schematic is shown in Figure 1. Our approach is similar to the optics solution in [8]. The image will be separated by energy using a spectrometer and then encoded by an aperture. Next, the spectral image will be recombined by a second spectrometer into an image. Between the two spectrometers, the spectral axis of the image will be tilted and exposed to the aperture, thus encoding each energy slice differently. The pixelated sensor detects the spectrally encoded image and compresses the spectral information at each spatial location into a single pixel. This approach could be also used with a single omega spectrometer.

Usually a multispectral image can be viewed as a cube with two spatial axes (axis $\mathrm{x}$ and axis $\mathrm{y}$ ) and one spectral axis (axis $z$ ). Given a spatial coordinate pair $(i, j)$, the spectral curve corresponds to one or a mixture of chemical elements. Our task can be summarized as classification of each coordinate $(i, j)$ to a particular element $(\mathrm{Sr}, \mathrm{Ti}, \mathrm{O})$ and possibly empty space. For each coordinate we compute the probability of being in each element class

$$
p_{c i j}=\exp \left\{-\left(\boldsymbol{y}_{i j}-\boldsymbol{\Phi}_{i j} \boldsymbol{s}_{c}\right)^{\top}\left(\boldsymbol{\Phi}_{i j} \boldsymbol{\Phi}_{i j}^{\top}\right)^{-1}\left(\boldsymbol{y}_{i j}-\boldsymbol{\Phi}_{i j} \boldsymbol{s}_{c}\right)\right\}
$$

where $\boldsymbol{y}_{i j}$ is the vector of encoded measurements, $\boldsymbol{\Phi}_{i j}$ represents the measurement matrix, and $\boldsymbol{s}_{c}$ is a reference spectra for element $c[3,4]$. The matrix $P_{c}$ can then be interpreted as an image where intensity indicates presence of element $c$ ( 0 means not present, 1 means present) [9]. 


\section{References:}

[1] A Stevens, L Kovarik, H Yang et al, Microscopy and Microanalysis 22(S3) (2016), pp. 560.

[2] A Stevens, L Kovarik, P Abellan et al. Adv. Structural and Chemical Imaging 1(10), (2015), pp. 1.

[3] A Stevens, H Yang, L Carin et al. Microscopy 63(1), (2014), pp. 41.

[4] D Mucke, P Abellan, M Sarahan et al, Microscopy \& Microanalysis 22(S3) (2016), pp. 558.

[5] L Kovarik, A Stevens, A Liyu et al. Appl. Phys. Lett. 109, 164102 (2016).

[6] M Davenport, P Boufounos, M Wakin et al, Sel. Top. in Sig. Proc., IEEE Jour. 4(2), (2010), pp. 445.

[7] H Reboredo, F Renna, R Calderban et al, Sig. Proc., IEEE Trans. 64(22), (2016), pp. 5778.

[8] P Poon, E Vera and M Gehm. Computational Imaging, SPIE 9870, 987003 (2016)

[9] Supported by the Chemical Imaging, Signature Discovery, and Analytics in Motion initiatives at PNNL. PNNL is operated by Battelle Memorial Inst. for the US DOE; contract DE-AC05-76RL01830.

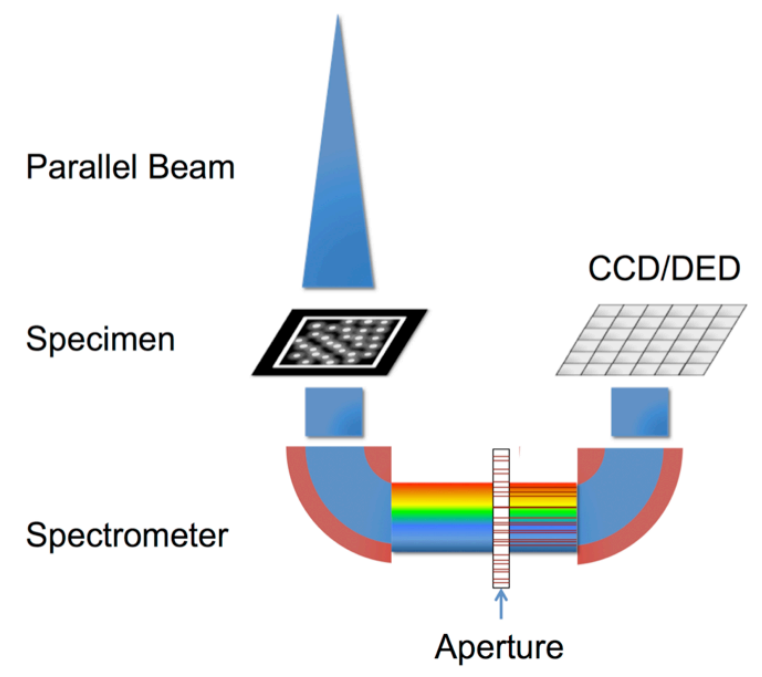

Figure 1. Schematic of TEM-EELS acquisition. The aperture or beam can be shifted to produce different encodings.

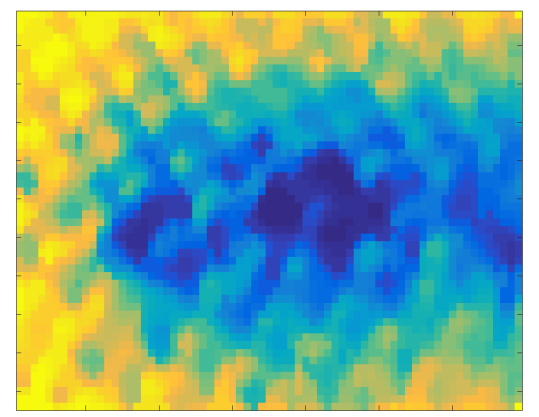

(a)

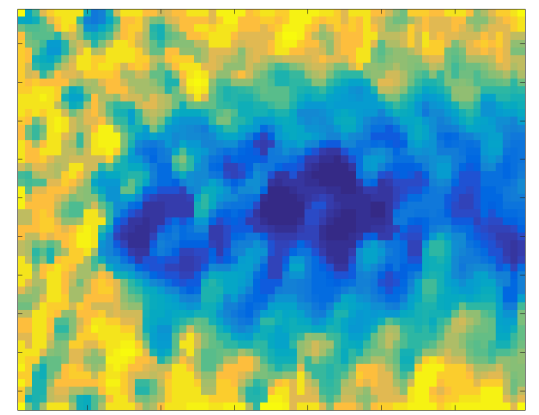

(b)

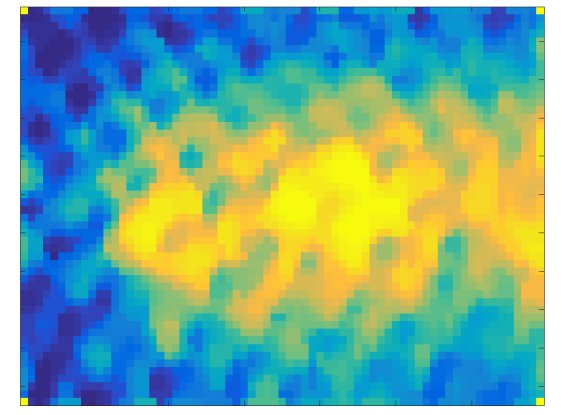

(c)

Figure 2. Elemental map for chemical elements. (a) is $\mathrm{Sr},(\mathrm{b})$ is $\mathrm{Ti}$, (c) is background. 